OPEN ACCESS

Edited by:

Robert Clarke,

Georgetown University, United States

Reviewed by:

James M. Rae

University of Michigan, United States John Hawse,

Mayo Clinic, United States

*Correspondence:

Yannick Goumon

yannick.goumon@inserm.u-strasbg.fr

Specialty section

This article was submitted to Pharmacology of Anti-Cancer Drugs,

a section of the journal

Frontiers in Oncology

Received: 24 September 2019 Accepted: 08 January 2020

Published: 21 February 2020

Citation:

Gabel F, Aubry A-S, Hovhannisyan V,

Chavant V, Weinsanto I, Maduna T,

Darbon P and Goumon Y (2020)

Unveiling the Impact of Morphine on

Tamoxifen Metabolism in Mice in vivo.

Front. Oncol. 10:25

doi: $10.3389 /$ fonc. 2020.00025

\section{Unveiling the Impact of Morphine on Tamoxifen Metabolism in Mice in vivo}

\author{
Florian Gabel ${ }^{1}$, Anne-Sophie Aubry ${ }^{1}$, Volodya Hovhannisyan ${ }^{1}$, Virginie Chavant ${ }^{1,2}$, \\ Ivan Weinsanto ${ }^{1}$, Tando Maduna ${ }^{1}$, Pascal Darbon ${ }^{1}$ and Yannick Goumon ${ }^{1,2 *}$ \\ ${ }^{1}$ CNRS UPR3212, Institut des Neurosciences Cellulaires et Intégratives, Centre National de la Recherche Scientifique, \\ University of Strasbourg, Strasbourg, France, ${ }^{2}$ Mass Spectrometry Facilities of the CNRS UPR3212, Institut des \\ Neurosciences Cellulaires et Intégratives, Centre National de la Recherche Scientifique, Strasbourg, France
}

Background: Tamoxifen is used to treat breast cancer and cancer recurrences. After administration, tamoxifen is converted into two more potent antitumor compounds, $4 \mathrm{OH}$-tamoxifen and endoxifen by the CYP3A4/5 and 2D6 enzymes in human. These active compounds are inactivated by the same UDP-glucuronosyltransferase isoforms as those involved in the metabolism of morphine. Importantly, cancer-associated pain can be treated with morphine, and the common metabolic pathway of morphine and tamoxifen suggests potential clinically relevant interactions.

Methods: Mouse liver microsomes were used to determine the impact of morphine on $4 \mathrm{OH}$-tamoxifen metabolism in vitro. For in vivo experiments, female mice were first injected with tamoxifen alone and then with tamoxifen and morphine. Blood was collected, and LC-MS/MS was used to quantify tamoxifen, $4 \mathrm{OH}$-tamoxifen, $\mathrm{N}$-desmethyltamoxifen, endoxifen, 4OH-tamoxifen-glucuronide, and endoxifen-glucuronide.

Results: In vitro, we found increased $K_{m}$ values for the production of $4 \mathrm{OH}$-tamoxifen-glucuronide in the presence of morphine, suggesting an inhibitory effect on $4 \mathrm{OH}$-tamoxifen glucuronidation. Conversely, in vivo morphine treatment decreased $4 \mathrm{OH}$-tamoxifen levels in the blood while dramatically increasing the formation of inactive metabolites $4 \mathrm{OH}$-tamoxifen-glucuronide and endoxifen-glucuronide.

Conclusions: Our findings emphasize the need for caution when extrapolating results from in vitro metabolic assays to in vivo drug metabolism interactions. Importantly, morphine strongly impacts tamoxifen metabolism in mice. It suggests that tamoxifen efficiency could be reduced when both drugs are co-administered in a clinical setting, e.g., to relieve pain in breast cancer patients. Further studies are needed to assess the potential for tamoxifen-morphine metabolic interactions in humans.

Keywords: Tamoxifen, 4OH-tamoxifen, endoxifen, Morphine, metabolism, CYP, UDP-glucuronosyltransferase, drug-drug interactions

\section{BACKGROUND}

Breast cancer is the most common and deadliest cancer diagnosed in women, even though major advances in screening and treatment have been made in the last 20 years (1). In estrogen receptor (ER)-positive breast tumors, the main strategy of breast anticancer drugs is to either antagonize ER signaling or decrease estrogen synthesis to prevent cancer cell proliferation. Among those drugs, 


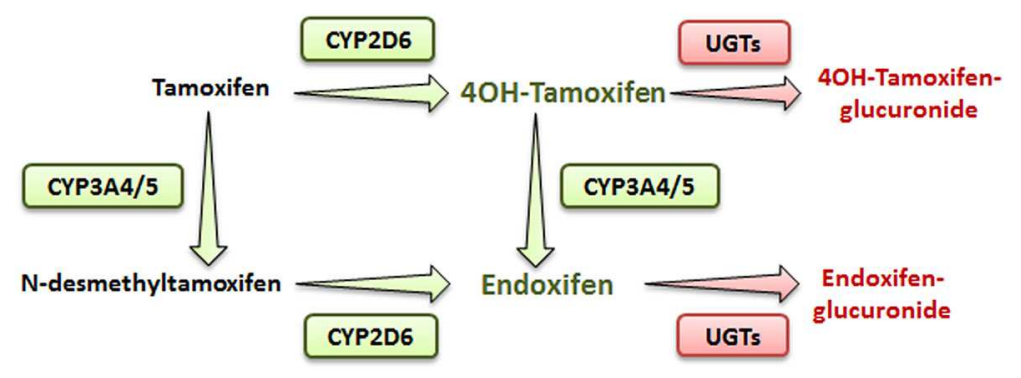

FIGURE 1 | Simplified metabolic pathway of tamoxifen in humans. Compounds in green and red are the active and inactive metabolites of tamoxifen, respectively.

tamoxifen is a selective estrogen receptor modulator (SERM) used for decades to decrease breast cancer recurrence (2). Nowadays, tamoxifen remains one of the major treatment for breast cancer, especially in countries with limited health care resources (3).

Tamoxifen is a pro-drug metabolized mostly in the liver by the phase I cytochrome P450 (CYP) 2D6 and 3A4/5 enzymes (4). In human, hydroxylation of tamoxifen (CYP2D6) leads to $4 \mathrm{OH}$-tamoxifen that can be further processed into endoxifen (via CYP3A4/5) through N-desmethylation (Figure 1). These two major metabolites are 30 - to 100 -fold more potent than tamoxifen itself and are responsible for its anti-tumoral activity.

In addition, $\mathrm{N}$-desmethylation of tamoxifen generates the $\mathrm{N}$-desmethyltamoxifen intermediate (CYP3A4/5) that is further metabolized into endoxifen through CYP2D6-mediated hydroxylation.

Endoxifen is the major metabolite of tamoxifen in humans. Alternatively, in mice, even though CYP2D6 isoform is absent, $4 \mathrm{OH}$-tamoxifen is the main anticancer product of tamoxifen, suggesting that other CYP2D isoforms, such as CYP2D22, could be involved in its metabolism $(4,5)$.

Phase II metabolizing enzymes including Uridine $5^{\prime}$ diphospho (UDP)-glucuronosyltransferases (UGT1A10, 1A4, 1A8, 2B7, and 2B15) convert active tamoxifen metabolites into inactive 4OH-tamoxifen-glucuronide and endoxifenglucuronide (Figure 1) (6). Approximately $75 \%$ of a given dose of tamoxifen is excreted into the biliary tract as inactive glucuronides (7).

Cancer-associated pain resulting from metastases, anticancer treatment or surgery represents a major problem that is treated with analgesic drugs including morphine, codeine, and/or paracetamol (8). Morphine remains the gold standard for moderate and severe pain relief despite side effects that limit its chronic use (9). In humans, morphine acts on $\mathrm{Mu}$ opioid receptors (MORs) to produce analgesia. Its metabolism in the

Abbreviations: ACN, acetonitrile; ADME, absorption, distribution, metabolism, and/or excretion; AF, formic acid; AI, aromatase-inhibitors; CYP, cytochrome P450; ER, estrogen receptor; i.p., injected intraperitoneally; IS, internal standard; LC-MS/MS, liquid chromatography-mass spectrometry/mass spectrometry; M3G, morphine-3-glucuronide; M6G, morphine-6-glucuronide; MOR, $\mathrm{Mu}$ opioid receptors; SEM, standard error of the mean; SERM, selective estrogen receptor modulator; TDM, therapeutic drug monitoring; UGT, UDP-glucuronosyltransferases; SULT, sulfotransferase. liver and brain leads mainly to the formation of morphine-3glucuronide (M3G) (10) and morphine-6-glucuronide (M6G) (10-12). In human, morphine-glucuronidation is catalyzed by UGT2B7 and to a lower extent by a number of other UGT isoforms (UGT1A10, UGT1A1, 1A3, 1A6, 1A8, 1A9, 2A1, and UGT2B21) $(9,13,14)$. However, in mice, UGT2B7 (the major enzyme involved in morphine metabolism in human) is absent but its activity is rescued by UGT2B21 and UGT2B36 (14-16).

Drug-drug interactions, resulting in either enzyme inhibition or induction, are a major limitation for the use of co-treatments (17). Usually, these drug-drug interactions are initially studied in vitro and then in vivo (18). While in vitro studies provide interesting results, their interpretation has proven to be complex when translated to in vivo drug metabolism (18).

Although anti-cancer agents share common catabolic pathways with many opiates, the impact of their coadministration on the metabolism and thus on the activity of anticancer drugs remains unexplored. These potential interactions between analgesic and anticancer drug metabolism could be used to treat more efficiently breast cancer. Therefore, as a proof of concept, we have investigated in mice whether morphine can alter tamoxifen metabolism.

\section{METHODS}

\section{Animals}

Experiments were performed with 11- to 29-week-old female C57BL/6J mice ( $23 \pm 4 \mathrm{~g}$; Charles River, L'Arbresle, France). Animals were housed according to a 12-h light-dark cycle, at a temperature of $22^{\circ} \mathrm{C} \pm 2{ }^{\circ} \mathrm{C}$ and provided with food and water ad libitum. All procedures were performed in accordance with European directives (2010/63/EU) and were approved by the regional ethics committee and the French Ministry of Agriculture (license no. APAFIS\#16827-2018092113192911 v4 to $Y G)$.

\section{Blood Collection}

The tail of the mouse was anesthetized locally with a cutaneous application of lidocaine/prilocaïne 5\% (Zentiva, Paris, France). After $5 \mathrm{~min}$, a small incision was performed at the end of the tail and $10 \mu \mathrm{l}$ of blood was collected using a 
calibrated capillary (Minicaps End-to-End $10 \mu \mathrm{l}$; Hischmann, Eberstadt Germany).

\section{Tamoxifen and Morphine Injections}

Female mice were injected intraperitoneally (i.p., calibrated Hamilton syringe) with $10 \mathrm{mg} / \mathrm{kg}$ of tamoxifen (in $90 \%$ olive oil/10\% ethanol, v/v; Sigma Aldrich, Lyon, France), and then with $\mathrm{NaCl} 0.9 \%$ at 0,1 , and $2 \mathrm{~h}$ following tamoxifen administration (Figure 2). Blood was collected by tail vein sampling (see above) just before and at $1,2,4,8,24$, and $48 \mathrm{~h}$ after tamoxifen injection (Figure 2). A second injection of tamoxifen was then performed at $48 \mathrm{~h}$ and immediately followed by an injection of either $10 \mathrm{mg} / \mathrm{kg}$ of morphine- $\mathrm{HCl}$ (diluted in $0.9 \% \mathrm{NaCl}$; Francopia, Paris, France) or saline solution $(0.9 \% \mathrm{NaCl}$ only). Mice then received two additional injections of morphine or saline at 1 and $2 \mathrm{~h}$ after the second tamoxifen dose. Blood was collected at 1, 2, 4, 8, 24, and $48 \mathrm{~h}$ after the second tamoxifen injection (Figure 2).

\section{Sample Preparation}

The blood was transferred from the capillary into a microtube containing $10 \mu \mathrm{l}$ of heparin and frozen at $-20^{\circ} \mathrm{C}$. On the next day, blood was thawed and $10 \mu \mathrm{l}$ of an internal standard (IS; see below) and $100 \mu \mathrm{l}$ of ice-cold acetonitrile (ACN; Thermo Scientific, San Jose, USA) were added. The samples were next vortexed and centrifuged at 20,000 g during $15 \mathrm{~min}$ at $4^{\circ} \mathrm{C}$. The supernatants were collected, dried under vacuum, and suspended in $15 \mu \mathrm{l}$ of $50 \%$ methanol/0.1\% formic acid (v/v; Sigma Aldrich) prior to LC-MS/MS analysis.

\section{Microsome Preparation}

Liver tissues were collected from 10-week-old male C57/BL6J mice. Samples were pooled and homogenized with an Ultra Turrax instrument (Ika, Staufen, Germany) in $10 \mathrm{ml}$ of extraction buffer (100 mM Na phosphate buffer, pH 7.4, 0.32 M sucrose, $1 \mathrm{mM}$ EDTA, $0.1 \mathrm{mM}$ DTT, protease inhibitor cOmplete Mini, EDTA-free, Roche, Basel, Switzerland). The homogenate was then sonicated $(2 \times 10 \mathrm{~s}, 100 \mathrm{~W})$ and centrifuged for $12 \mathrm{~min}$ at $2,000 \mathrm{~g}\left(4^{\circ} \mathrm{C}\right)$. The supernatant was transferred into polycarbonate ultracentrifuge tubes (Beckman Instruments, Palo Alto, USA), completed with extraction buffer and centrifuged $40 \mathrm{~min}$ at $10,000 \mathrm{~g}$ and $4^{\circ} \mathrm{C}$ in a type-70 Ti Rotor (Beckman Coulter, Brea, USA). The resulting supernatant was then centrifuged for $130 \mathrm{~min}$ at $130,000 \mathrm{~g}\left(4^{\circ} \mathrm{C}\right)$, and the pellet obtained was suspended in $800 \mu \mathrm{l}$ of storage buffer $(100 \mathrm{mM}$ Na phosphate buffer, pH 7.4, 0.5 mM EDTA, 0.1 mM DTT, 20\% glycerol; Sigma Aldrich) and frozen. Protein concentration was determined using the Bradford method (Protein Assay, Bio-Rad, Marnes-la-Coquette, France).

\section{Enzymatic Activity Assay}

One hundred micrograms of liver microsomes were used to perform 4OH-tamoxifen glucuronidation assays. First, increasing concentrations of $4 \mathrm{OH}$-tamoxifen $(10,20,40$, $50,60,70,80,100,125,150,200,250$, and $300 \mu \mathrm{M}$; LGC Standard, Molsheim, France) with a fixed concentration of morphine $(500 \mu \mathrm{M})$ were dried under vacuum. Morphine was suspended in $4 \mathrm{mM} \mathrm{MgCl}_{2}$ adjusted with $\mathrm{H}_{2} \mathrm{O}$, and each $4 \mathrm{OH}$-tamoxifen concentration was diluted with $69 \mu \mathrm{l}$ of the morphine-containing mix.

Microsomes were incubated for $15 \mathrm{~min}$ at $4^{\circ} \mathrm{C}$ in the presence of alamethicin $(30 \mu \mathrm{g} / \mathrm{mg}$ of protein; Santa Cruz Biotechnology, Heidelberg, Germany) and Tris- $\mathrm{HCl}$ buffer ( $400 \mathrm{mM})$ adjusted with $\mathrm{H}_{2} \mathrm{O}$. Then, $75 \mu \mathrm{l}$ of microsome were added to each $4 \mathrm{OH}-$ tamoxifen concentration and tubes were equilibrated at $37^{\circ} \mathrm{C}$ during $5 \mathrm{~min}$. The enzymatic reactions were started with the addition of $6 \mu \mathrm{l}$ of UDPGA to a final concentration of $5 \mathrm{mM}$. Reactions were stopped $20 \mathrm{~s}$ later with $900 \mu \mathrm{l}$ of cold $100 \%$

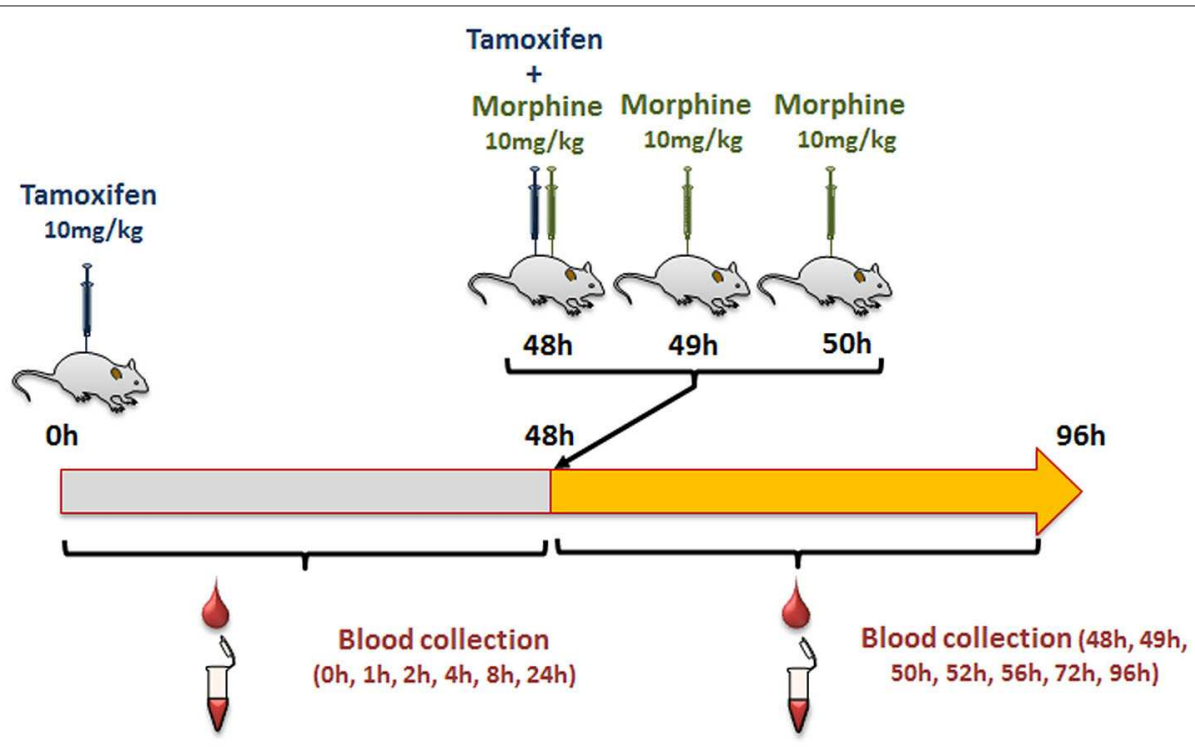

FIGURE 2 | Protocol used to study tamoxifen-morphine drug-drug interactions. 
methanol. Samples were then diluted (1:5), and an IS (see below) was added to each sample. Samples were centrifuged for $15 \mathrm{~min}$ at $20,000 \mathrm{~g}$, and $4^{\circ} \mathrm{C}$ and the supernatants were dried under vacuum and then suspended in $45 \mu \mathrm{l}$ of $50 \%$ methanol/0.1\% formic acid (v/v) prior to LC-MS/MS analysis. $K_{\mathrm{m}}$ and $V_{\max }$ were obtained with a Michaelis-Menten plot following a non-linear curve fit with the least-squares method (GraphPad Prism 6 software).

\section{LC-MS/MS Instrumentation and Analytical Conditions}

Analyses were performed with a Dionex Ultimate 3000 HPLC system (Thermo Scientific) coupled with a triple quadrupole Endura mass spectrometer. Xcalibur v2.0 software was used to control the system (Thermo Electron, Villebon Sur Yvette, France). Samples were loaded onto an Accucore RP-MS column $(150 \times 1 \mathrm{~mm}, 2 \mu \mathrm{m}$, flow of $90 \mu \mathrm{l} / \mathrm{min}$; Thermo Electron) heated at $40^{\circ} \mathrm{C}$. Buffer A was $1 \% \mathrm{ACN} / 98.9 \% \mathrm{H}_{2} \mathrm{O} / 0.1 \%$ formic acid (v/v/v), whereas buffer B was $99.9 \%$ ACN/0.1\% formic acid (v/v). The gradient used is detailed in Supplementary Table 1.

Electrospray ionization was achieved in the positive mode with the spray voltage set at 3,500 V. Nitrogen was used as the nebulizer gas, and the ionization source was heated to $250^{\circ} \mathrm{C}$. Desolvation (nitrogen) sheath gas was set to 18 Arb and Aux gas was set to $7 \mathrm{Arb}$. Ion transfer tube was heated at $297^{\circ} \mathrm{C}$. Q1 and Q2 resolutions were set at 0.7 FWHM, whereas collision gas (CID, argon) was set to 2 mTorr. Identification of the compounds was based on precursor ion, selective fragment ions, and retention times. Selection of the monitored transitions and optimization of collision energy and RF Lens parameters were manually determined (see Supplementary Table 1 for details). Qualification and quantification were performed in MRM mode using Quan Browser software (Thermo Scientific).

\section{Statistics}

Statistical analysis was performed using GraphPad Prism 6 Software. Results were presented as mean values \pm standard error of the mean (SEM). Groups were compared using multiple $t$-tests.

\section{RESULTS}

\section{Enzymatic Study in vitro}

As $4 \mathrm{OH}$-tamoxifen is the major active metabolite of tamoxifen in mice, in vitro experiments were performed on mouse liver microsomes to study the impact of $500 \mu \mathrm{M}$ of morphine on the glucuronidation of $4 \mathrm{OH}$-tamoxifen. Morphine was used at $500 \mu \mathrm{M}$ to determine the $K_{\mathrm{m}}$ of the glucuronidation of $4 \mathrm{OH}$-tamoxifen as this concentration corresponds to the $K_{\mathrm{m}}$ previously determined for morphine glucuronidation in mice $(12,19)$. As shown in Figure 3, morphine significantly affects the production of $4 \mathrm{OH}$-tamoxifen-glucuronide. Specifically, morphine significantly reduced the production of $4 \mathrm{OH}$ tamoxifen-glucuronide when 10 to $50 \mu \mathrm{M}$ and $70 \mu \mathrm{M}$ of tamoxifen were used. $K_{\mathrm{m}}$ values for the production of $4 \mathrm{OH}$ tamoxifen-glucuronide in the absence and presence of morphine, as determined by the Michaelis-Menten equation, were 68 and $98.6 \mu \mathrm{M}(+45 \%)$, respectively.

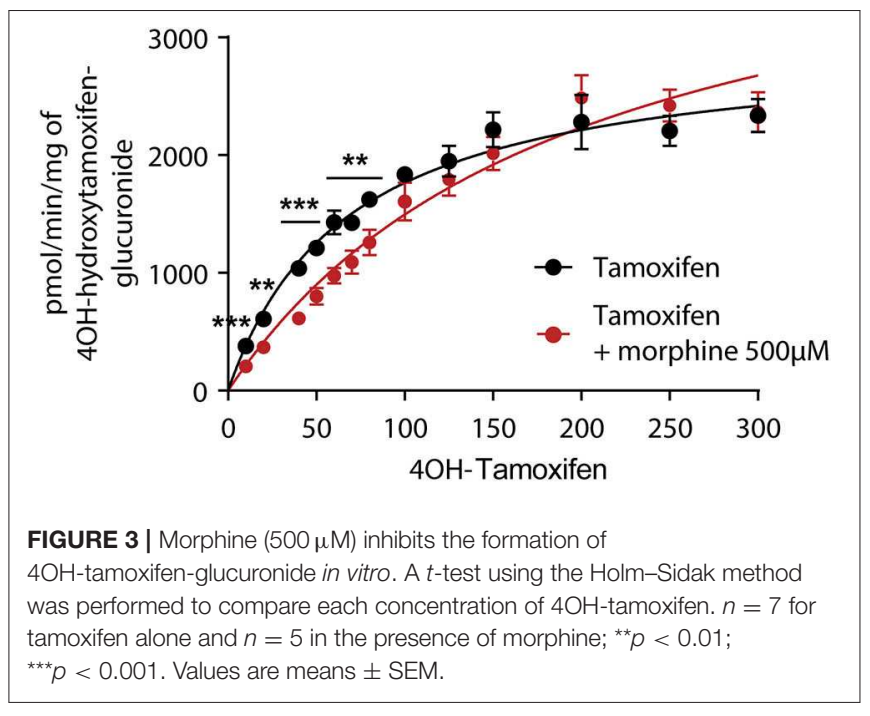

These results indicate that morphine reduces $4 \mathrm{OH}$-tamoxifen glucuronidation in vitro.

\section{Study of Tamoxifen Metabolism in vivo}

First, we determined whether multiple injections of tamoxifen would alter its own metabolism (Figures 2, 4A). Blood was collected before and $1,2,4,8,24$, and $48 \mathrm{~h}$ after the first (Figure 4A, white part) and the second injection of tamoxifen (Figure 4A, gray part). Tamoxifen, 4OH-tamoxifen, and endoxifen-glucuronide concentrations in the blood did not vary significantly at any time point between the two tamoxifen injections (Figure 4B). In contrast, a significant increase in the concentrations of $4 \mathrm{OH}$-tamoxifen-glucuronide, $\mathrm{N}$ desmethyltamoxifen and endoxifen was observed. Accordingly, drug metabolic ratios (i.e., the concentration ratio of a metabolite compared to its parent molecule) were significantly altered at different time points (Figures 5A-F). The ratio of endoxifen/N-desmethyltamoxifen was significantly elevated at 4 and $8 \mathrm{~h}$ compared to the first injection suggesting an increase in endoxifen synthesis (Figure 5C). In a more dramatic manner, 4OH-tamoxifen glucuronidation was increased by $1.5-$ to 2-fold at all time points compared to the first injection (Figure 5F). Similarly, the $t=2 \mathrm{~h}$ ratio of endoxifen-glucuronide to its parent molecule endoxifen showed a 3 -fold increase compared to the first injection (Figure 5E). On the other hand, no difference was observed for $4 \mathrm{OH}$-tamoxifen/tamoxifen (Figure 5A), N-desmethyltamoxifen/tamoxifen (Figure 5B), and endoxifen/4OH-tamoxifen ratios (Figure 5D). Together, these results indicate that tamoxifen metabolism is slightly potentiated following two subsequent injections of the drug.

As morphine has a short half-life in mice $(30 \mathrm{~min})$, we have performed three injections of morphine to reach adequate concentrations in the blood (Supplementary Figure 1). The highest concentrations of morphine and M3G in the blood were reached after $2 \mathrm{~h}(1,599 \pm 336 \mathrm{pmol} / \mathrm{ml}$ and 9,773 $\pm 1,274$ $\mathrm{pmol} / \mathrm{ml}$, respectively). Morphine was still present after $8 \mathrm{~h}$, allowing a long-lasting competition with tamoxifen metabolism. 

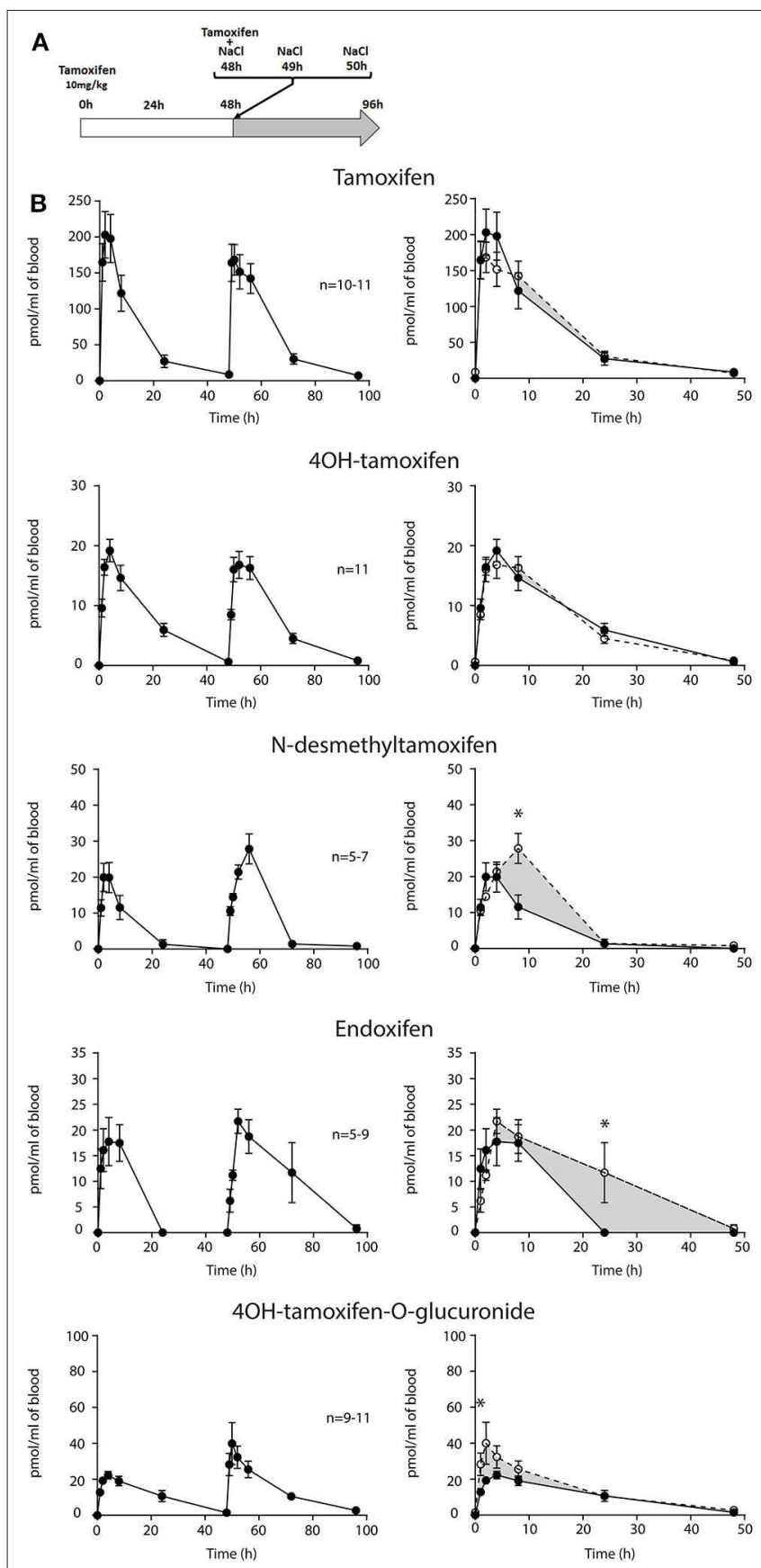

Endoxifen-glucuronide
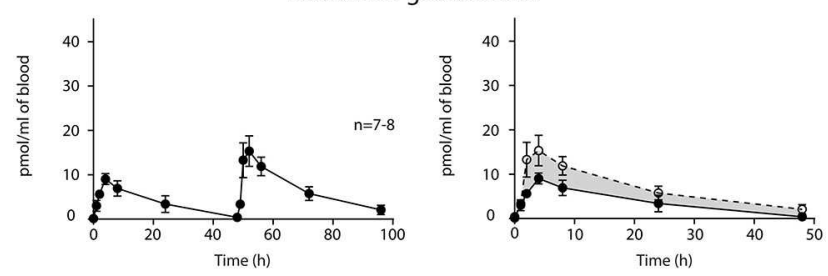

FIGURE 4 | Tamoxifen metabolism is affected by a prior injection. Effect of two subsequent injections of tamoxifen (10 mg/kg i.p.) on the levels of tamoxifen and its metabolites. (A) Protocol. Injections of $\mathrm{NaCl} 0.9 \%$ at $\mathrm{Oh}, 1 \mathrm{~h}$, and $2 \mathrm{~h}$ are not represented. (B) Left panels, levels of tamoxifen, $4 \mathrm{OH}$-tamoxifen,

(Continued)
FIGURE 4 | N-desmethyltamoxifen, endoxifen, 4OH-tamoxifen-glucuronide, and endoxifen-glucuronide during $96 \mathrm{~h}$. Right panels correspond to the superimposition of the first 0-48 h (white area) and last 48-96 h (gray area). The gray area corresponds to an increase in the quantity of the corresponding molecule after the second injection (48-96 h). Multiple $t$-tests with the Holm-Sidak correction were applied. Values are means \pm SEM. ${ }^{*} p<0.05$.

Then, female mice were injected twice with tamoxifen (at 0 and $48 \mathrm{~h}$ ) in addition to morphine (at 48,49 , and $50 \mathrm{~h}$ ) and blood samples were collected (Figure 6A). Following morphine injections, the blood concentrations of tamoxifen, 4OH-tamoxifen, 4OH-tamoxifen-glucuronide, endoxifen, and endoxifen-glucuronide were significantly increased compared to the first injection of tamoxifen (Figure 6B). Only a tendency was observed for $\mathrm{N}$-desmethytamoxifen. More importantly, ratios between $4 \mathrm{OH}$-tamoxifen/tamoxifen (Figure 7A) were significantly decreased by $1 / 2$ - to $1 / 5$-fold 1,2 , and $8 \mathrm{~h}$ after the injection of morphine, suggesting that $4 \mathrm{OH}$-tamoxifen was processed into its metabolites at a faster rate in the presence of morphine. Indeed, the ratios of $4 \mathrm{OH}$-tamoxifenglucuronide/4OH-tamoxifen showed a significant increase (2- to 3-fold) at every time point (Figure 7F). Similarly, endoxifen-glucuronide/endoxifen ratios (Figure 7E) were dramatically increased (1.5- to 4 -fold) at 2,4 , and $8 \mathrm{~h}$ after the injection of morphine. On the other hand, the ratios of $\mathrm{N}$-desmethyltamoxifen/tamoxifen (Figure 7B), endoxifen/Ndesmethyltamoxifen (Figure 7C) and endoxifen/4OH-tamoxifen (Figure 7D) were not altered by morphine administration. Together, these results indicate that the inactivation of tamoxifen and its active metabolites is exacerbated in the presence of equimolar amounts of morphine.

\section{DISCUSSION}

\section{Repeated Tamoxifen Treatment Potentiates Glucuronide Formation in vivo}

Our results show that the blood formation pattern of $\mathrm{N}$ desmethyltamoxifen and endoxifen is slightly modified in vivo after two subsequent tamoxifen treatments. Indeed, we observed a higher peak concentration in the case of $\mathrm{N}$ desmethyltamoxifen and a slower elimination for endoxifen upon the second administration of tamoxifen. Furthermore, analysis of metabolic ratios revealed an increase in $4 \mathrm{OH}-$ tamoxifen-glucuronide and endoxifen-glucuronide formation compared to their parent drugs when animals received a second injection of tamoxifen. Such an increase of glucuronidation can be related to induction of the expression of UGTs present in the liver occurring $48 \mathrm{~h}$ after the first injection of tamoxifen. Indeed, it has been described that several xenobiotics are able to promote UGT expression by acting on regulatory elements in the cell (20). Tamoxifen acts as a selective modulator on the ER, which, in turn, modulates the activity of numerous transcription factors implicated in the regulation of gene expression. Importantly, tamoxifen has been shown to increase the expression of CYP enzymes involved in its own metabolism, such as CYP3A4 (21). In the same manner, 

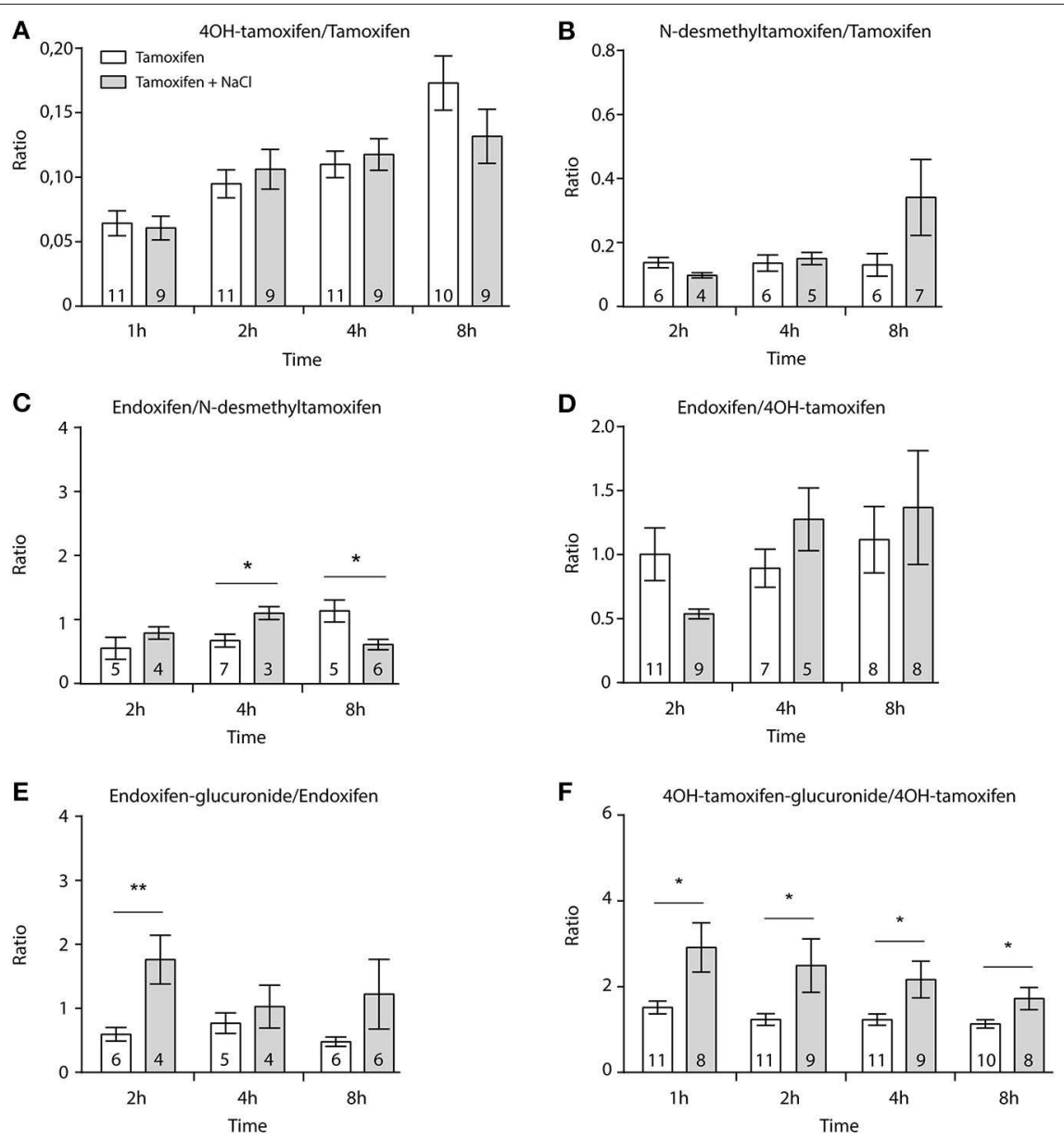

FIGURE 5 | Tamoxifen potentiates its own metabolism. Ratio between metabolites and parent compounds. (A) 4OH-tamoxifen/tamoxifen, (B) $\mathrm{N}$-desmethyltamoxifen/tamoxifen, (C) endoxifen/N-desmethyltamoxifen, (D) endoxifen/4OH-tamoxifen, (E) endoxifen-glucuronide/endoxifen, and (F) $4 \mathrm{OH}$-tamoxifen-glucuronide/4OH-tamoxifen. $N$ are indicated within columns. Values are means $\pm \mathrm{SEM}$. $t$-tests; ${ }^{*} p<0.05$; ${ }^{\star *} p<0.001$.

one may hypothesize that the first injection of tamoxifen induced the expression of UGTs, resulting in a potentiation of $4 \mathrm{OH}$-tamoxifen and endoxifen glucuronidation upon the second treatment.

Surprisingly, despite an increase in tamoxifen glucuronidation, we observed no concurrent decrease in the concentrations of $4 \mathrm{OH}$-tamoxifen or endoxifen. The main degradation pathway of tamoxifen is glucuronidation, but significant amounts of its two active metabolites are eliminated through sulfation. Several sulfotransferase (SULT) isoforms (1A1, 1E1, and 2A1) have been implicated in the degradation of $4 \mathrm{OH}$-tamoxifen (22). In addition, it has been shown in vitro that tamoxifen metabolites are able to inhibit SULT2A1 through mixed or non-competitive inhibition (23). Therefore, it is possible that our first tamoxifen administration inhibited SULT expression toward 4OH-tamoxifen and endoxifen. Thus, the balance between glucuronidation and sulfation could be modified without affecting $4 \mathrm{OH}$-tamoxifen or endoxifen levels. Nevertheless, this hypothesis remains to be tested.

\section{Morphine Increases Glucuronidation of Tamoxifen Active Metabolites}

Morphine was expected to reduce the glucuronidation of tamoxifen active metabolites through direct competition on the UGT-binding site as observed in vitro. Surprisingly, our results showed a dramatic increase in the levels of all active and inactive metabolites of tamoxifen when morphine was co-administered. The significant elevated levels of tamoxifen found in the blood after the coinjection with morphine may explain the increase observed for all compounds. This increase is likely to rely on differences of absorption due to drug-drug interactions with morphine rather than variability in tamoxifen injections. This point is strengthened by the fact that 19 mice were injected using a calibrated Hamilton syringe. Ratio between metabolites and their corresponding parent molecules were established to normalize the metabolite production with the tamoxifen injections. Analysis of the ratio revealed that morphine dramatically decreased the amount of $4 \mathrm{OH}$-tamoxifen relative to that of its prodrug in the blood of tamoxifentreated mice. This decrease is likely related to the concurrent 

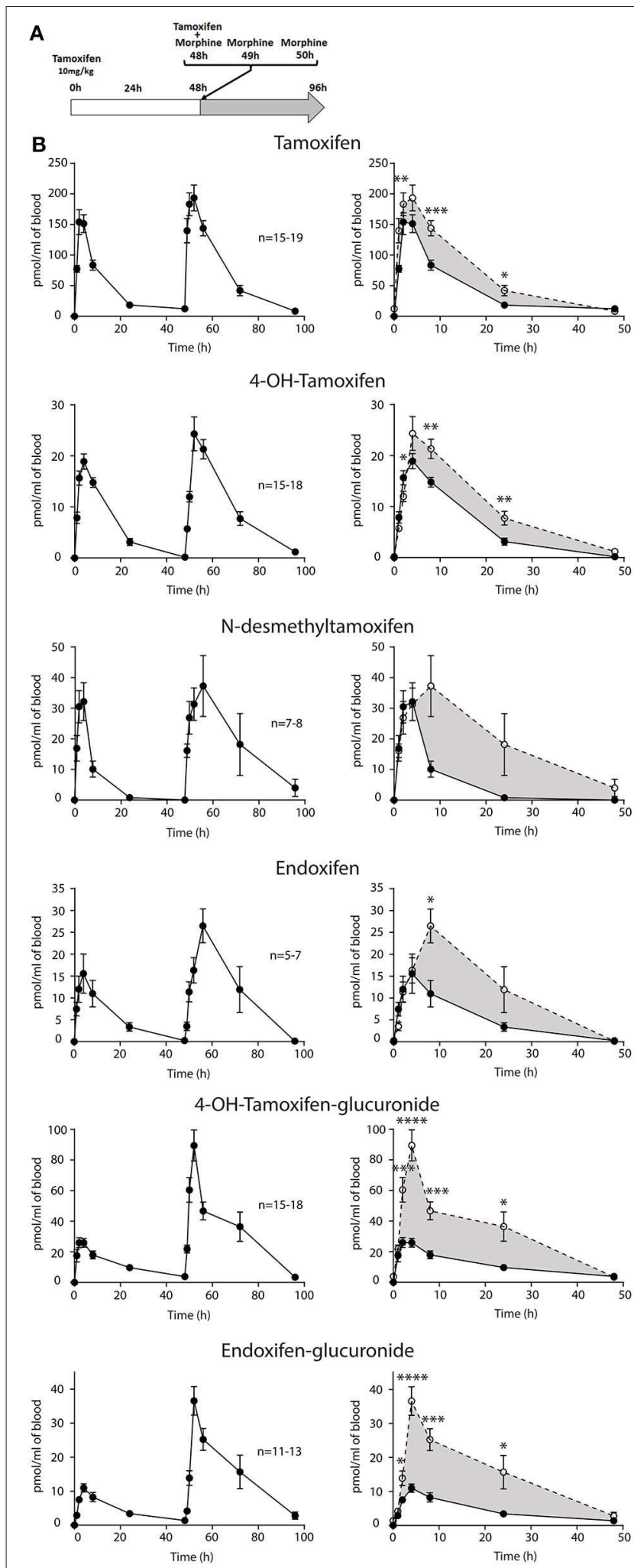

FIGURE 6 | Morphine increases tamoxifen metabolism. Effect of three injections of morphine $(10 \mathrm{mg} / \mathrm{kg}$ i.p.) on the blood concentrations of tamoxifen, $4 \mathrm{OH}$-tamoxifen, $\mathrm{N}$-desmethyltamoxifen, endoxifen, 4OH-tamoxifen-glucuronide, and endoxifen-glucuronide. (A) Protocol.

(Continued)
FIGURE 6 | Injections of $\mathrm{NaCl} 0.9 \%$ at 0, 1 , and $2 \mathrm{~h}$ are not represented. (B) Left panels, levels of tamoxifen, $4 \mathrm{OH}$-tamoxifen, $\mathrm{N}$-desmethyltamoxifen, endoxifen, $4 \mathrm{OH}$-tamoxifen-glucuronide, and endoxifen-glucuronide during $96 \mathrm{~h}$. Right panels correspond to the superimposition of the first 0-48 h (white area) and last 48-96 h (gray area). Gray area corresponds to an increase of the quantity of the corresponding molecule after the second injection (48-96 h). Values are means \pm SEM. ${ }^{\star} p<0.05 ;{ }^{* \star} p<0.01 ;{ }^{* \star} p<0.001$.

massive increase of the glucuronidation of $4 \mathrm{OH}$-tamoxifen and endoxifen.

It seems improbable that morphine would act as a cofactor of UGTs, allowing faster glucuronidation since it did not occur in our in vitro experiments. A potential impact of morphine on the entry of tamoxifen in hepatocytes is also unlikely because tamoxifen is known to cross the cell membrane passively (7), whereas morphine influx relies on transporters including organic cation transporter 1 (OCT1) (24). The last type of common molecular targets in the metabolism of tamoxifen and morphine are MRP (multidrug resistance-associated protein) and MDR (multidrug resistant protein) transporters driving M3G, 4OH-tamoxifen, endoxifen, 4OH-tamoxifen-glucuronide, and endoxifen-glucuronide out of the cell $(7,16,25,26)$. One hypothesis involving those transporters may be that morphine decreases the efflux rate of tamoxifen active metabolites (and thus increases their glucuronidation rate). Additional studies are needed to decipher the molecular mechanism underlying this atypical change in tamoxifen metabolism.

In conclusion, co-administration of morphine in mice appears to promote the inactivation of the potent $4 \mathrm{OH}$-tamoxifen and endoxifen metabolites. In light of these findings, we hypothesize that morphine could reduce the potency of tamoxifen anticancer treatment in mice. Further studies should determine if the impact of morphine on tamoxifen metabolism is sufficient to result in changes in anticancer activity at therapeutic doses.

\section{Strengths and Limitations}

We chose to associate morphine with tamoxifen to develop our methodology as it was expected to be a simple model focusing primarily on the glucuronidation process. Morphine is mainly metabolized by UGTs and was not expected to impact CYP activity. Morphine and tamoxifen co-treatments are given after surgeries or in the case of severe cancer pain (27). Otherwise, codeine and/or paracetamol are widely prescribed (8). In human, these two compounds are metabolized by the same CYPs (6D6/3A4) and UGTs (1A10, 1A4, 1A8, 2B7, and 2B15) $(28,29)$ as tamoxifen and might have a more complex impact on tamoxifen metabolic pathways $(30,31)$.

A main limitation of our study is that tamoxifen and morphine metabolisms differ in mice compared to humans. $4 \mathrm{OH}$-tamoxifen is the major active mouse metabolite whereas endoxifen is found at greater concentrations in human serum. However, our approach using the isotopic dilution allowed us to observe non-negligible levels of both endoxifen and endoxifen-glucuronide in the blood of tamoxifen-treated mice. In mice, the Cyp2d gene cluster displays nine functional genes 

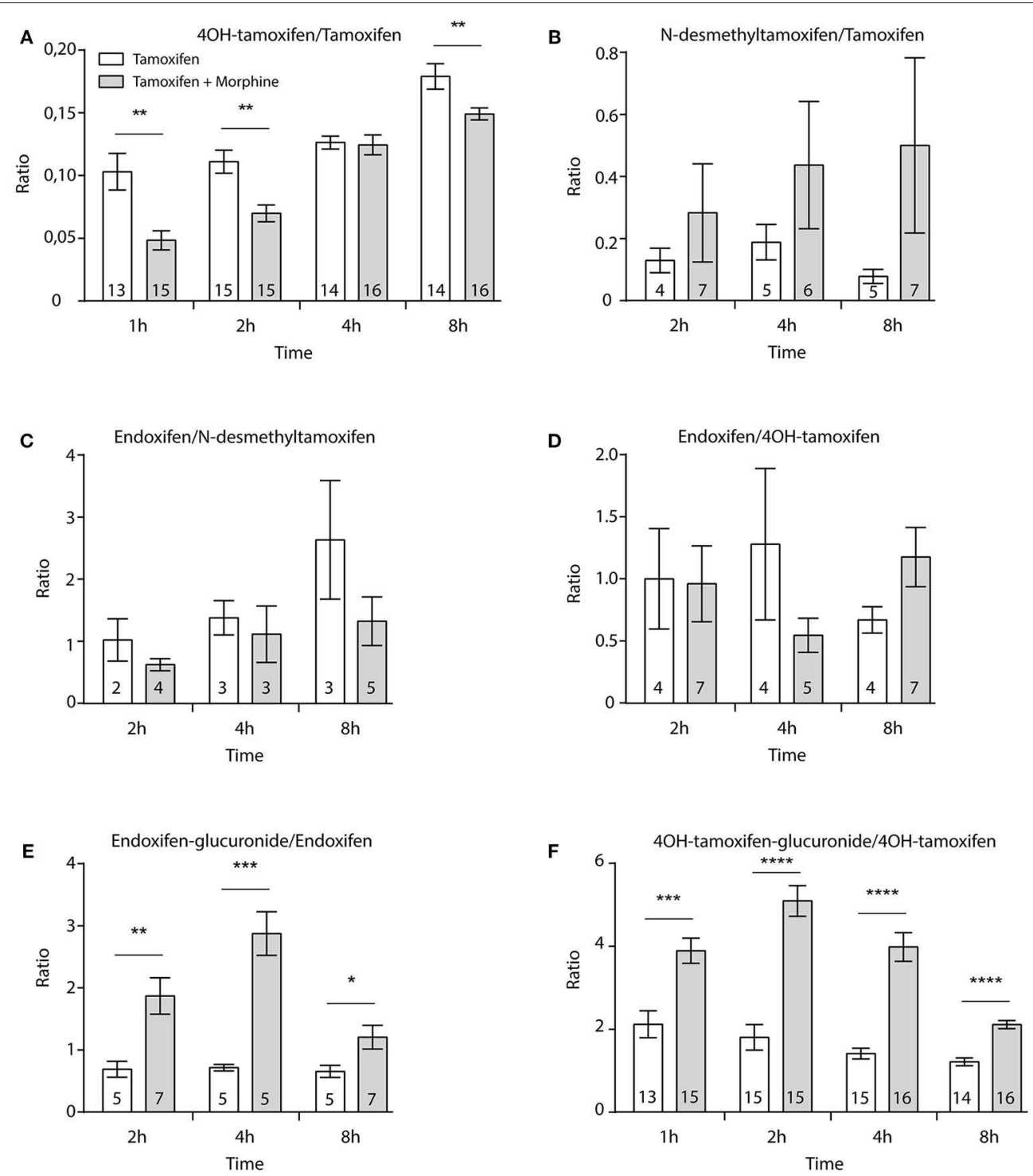

FIGURE 7 | Morphine promotes the inactivation of tamoxifen through increased glucuronidation. Effect of three injections of morphine (10 mg/kg i.p.) on the ratio between metabolites and parent compounds. (A) 4OH-tamoxifen/tamoxifen, (B) N-desmethyltamoxifen/tamoxifen, (C) endoxifen/ $\mathrm{N}$-desmethyltamoxifen, (D) endoxifen/4OH-tamoxifen, (E) endoxifen-glucuronide/endoxifen, and (F) $4 \mathrm{OH}$-tamoxifen-glucuronide/4OH-tamoxifen. $\mathrm{N}$ are indicated within columns. $t$-tests; ${ }^{*} p<0.05 ;{ }^{* *} p<0.01 ;{ }^{* \star *} p<0.001 ;{ }^{* * * *} p<0.0001$.

(Cyp2d9, Сyp2d10, Cyp2d11, Сyp2d12, Сyp2d13, Сyp2d22, Cyp2d26, Cyp2d34, and Cyp2d40), whereas humans only have one (CYP2D6) (4). Therefore, the presence of endoxifen suggests that CYP2D6 activity is rescued by an alternative CYP.

In addition, morphine is only metabolized into M3G in mice vs. M3G and M6G in humans (32, 33). Nevertheless, both species eliminate tamoxifen and morphine predominantly through glucuronidation. UGT2B7 (15), the main UGT involved in morphine metabolism in humans, is absent in mice. However, morphine and tamoxifen glucuronidation could be compensated by other enzymes including the mouse homologs of human UGT2B6, 2C9, 2C19, 3A4/5 (34), UGT2B36, and UGT2B21 $(14,15)$. These differences lead to a tamoxifen half-life of
$27 \mathrm{~h}$ in humans and $6.8 \mathrm{~h}$ in mice (4), as well as a morphine half-life of $30 \mathrm{~min}$ in mice and $2 \mathrm{~h}$ in humans $(32,33)$. Despite the existence of mouse equivalents to human CYP and UGT isoforms, major differences in isoform sequence and expression patterns limit the extrapolation of mouse data to humans. The development of humanized mouse models for CYP and UGT genes will allow overcoming such issues $(34,35)$.

Drug-drug interactions can lead to severe adverse effects and predicting these interactions in vivo is challenging. Thus, the Food and Drug Administration (FDA) and European Medicines Agency (EMA) are frequently publishing new guidelines regarding in vitro and in vivo drug-drug interaction 
studies (36). We have used an in vivo methodology to monitor modulations of tamoxifen metabolism. Intraperitoneal injections of tamoxifen were used instead of oral administration (the typical route of administration in humans) in order to better control the given amounts of tamoxifen and morphine (37). Indeed, the most used method is intraperitoneal injection, because the amount of administered compound can be better controlled, but delivery by oral gavage is also possible. However, oral administration suffers from significant first-pass metabolism (38), which limits absorption (39) and introduces inter-individual variability in drug metabolism (40). The pharmacokinetics of tamoxifen were obtained by quantification of tamoxifen and its metabolites following an initial injection $(10 \mathrm{mg} / \mathrm{kg})$. Then, a second injection was used to determine its pharmacokinetics in the absence or the presence of the competing drug morphine. Therefore, it was possible to accurately compare tamoxifen pharmacokinetics in the same animal to assess its potential interaction with morphine in vivo. It is however important to determine whether an injection of the drug of interest can induce adaptive processes responsible for differences in its metabolism following a second injection or chronic treatment.

\section{CONCLUSIONS}

In this study, we have investigated the effects of morphine on tamoxifen metabolism in vitro and in vivo. We have shown that in vitro morphine inhibits $4 \mathrm{OH}$-tamoxifen glucuronidation. Conversely, morphine reduced the blood levels of $4 \mathrm{OH}-$ tamoxifen in mice, while the inactivation of tamoxifen active compounds through glucuronidation greatly increased.

Our results suggest that morphine co-treatment could dramatically affect tamoxifen efficacy and emphasize the need to test more common analgesics (e.g., codeine or paracetamol) in humans to re-evaluate the impact of pain treatments on anti-cancer drug metabolism and pharmacological activity.

\section{REFERENCES}

1. Harbeck N, Penault-Llorca F, Cortes J, Gnant M, Houssami N, Poortmans P, et al. Breast cancer. Nat Rev Dis Primers. (2019) 5:66. doi: 10.1038/s41572-019-0111-2

2. Gail MH, Costantino JP, Bryant J, Croyle R, Freedman L, Helzlsouer K, et al. Weighing the risks and benefits of tamoxifen treatment for preventing breast cancer. J Natl Cancer Inst. (1999) 91:1829-46. doi: 10.1093/jnci/91.21.1829

3. Kantelhardt EJ, Hanson C, Albert US, Wacker J. Breast cancer in countries of limited resources. Breast Care. (2008) 3:10-6. doi: 10.1159/000114409

4. Reid JM, Goetz MP, Buhrow SA, Walden C, Safgren SL, Kuffel MJ, et al. Pharmacokinetics of endoxifen and tamoxifen in female mice: implications for comparative in vivo activity studies. Cancer Chemother Pharmacol. (2014) 74:1271-8. doi: 10.1007/s00280-014-2605-7

5. Blume $\mathrm{N}$, Leonard $\mathrm{J}$, $\mathrm{Xu} \mathrm{ZJ}$, Watanabe $\mathrm{O}$, Remotti $\mathrm{H}$, Fishman J. Characterization of Cyp2d22, a novel cytochrome P450 expressed in mouse mammary cells. Arch Biochem Biophys. (2000) 381:191-204. doi: 10.1006/abbi.2000.1978

6. Wu B, Kulkarni K, Basu S, Zhang S, Hu M. First-pass metabolism via UDPglucuronosyltransferase: a barrier to oral bioavailability of phenolics. J Pharm Sci. (2011) 100:3655-81. doi: 10.1002/jps.22568

\section{DATA AVAILABILITY STATEMENT}

The datasets generated for this study are available on request to the corresponding author.

\section{ETHICS STATEMENT}

All animal procedures were performed in accordance with European directives (2010/63/EU) and were approved by the regional ethics committee and the French Ministry of Agriculture (license No. APAFIS\#16827-2018092113192911 v4 to YG).

\section{CONSENT FOR PUBLICATION}

All authors have approved the manuscript for submission.

\section{AUTHOR CONTRIBUTIONS}

Conceptualization: FG, VC, IW, and YG. Methodology: FG, VC, IW, PD, and YG. Investigation: FG, VC, IW, VH, TM, and A-SA. Writing-original draft: YG, FG, and PD. Writing-review and editing: YG, FG, A-SA, VH, TM, and PD. Funding acquisition, resources, and supervision: $\mathrm{YG}$.

\section{FUNDING}

This work was funded by Fondation Alsace Contre le Cancer (YG), ITMO Cancer (YG), Inserm (YG), CNRS (YG), University of Strasbourg (YG), and Ministère Délégué à la Recherche et à l'Enseignement Supérieur (Ph.D. fellowship to IW and FG).

\section{SUPPLEMENTARY MATERIAL}

The Supplementary Material for this article can be found online at: https://www.frontiersin.org/articles/10.3389/fonc. 2020.00025/full\#supplementary-material

7. Klein DJ, Thorn CF, Desta Z, Flockhart DA, Altman RB, Klein TE. PharmGKB summary: tamoxifen pathway, pharmacokinetics. Pharmacogenet Genomics. (2013) 23:643-7. doi: 10.1097/FPC.0b013e3283656bc1

8. Lee SK, Dawson J, Lee JA, Osman G, Levitin MO, Guzel RM, et al. Management of cancer pain: 1. Wider implications of orthodox analgesics. Int J Gen Med. (2014) 7:49-58. doi: 10.2147/IJG M.S42187

9. Laux-Biehlmann A, Mouheiche J, Veriepe J, Goumon Y. Endogenous morphine and its metabolites in mammals: history, synthesis, localization and perspectives. Neuroscience. (2013) 233:95-117. doi: 10.1016/j.neuroscience.2012.12.013

10. Grace PM, Ramos KM, Rodgers KM, Wang X, Hutchinson MR, Lewis MT, et al. Activation of adult rat CNS endothelial cells by opioid-induced Toll-like receptor 4 (Tlr4) signaling induces proinflammatory, biochemical, morphological, and behavioral sequelae. Neuroscience. (2014) 280:299317. doi: 10.1016/j.neuroscience.2014.09.020

11. Lotsch J, Geisslinger G. Morphine-6-glucuronide: an analgesic of the future? Clin Pharmacokinet. (2001) 40:48599. doi: 10.2165/00003088-200140070-00001

12. Weinsanto I, Laux-Biehlmann A, Mouheiche J, Maduna T, Delalande F, Chavant V, et al. Stable isotope-labelled morphine to study in vivo central and 
peripheral morphine glucuronidation and brain transport in tolerant mice. $\mathrm{Br}$ J Pharmacol. (2018) 175:3844-56. doi: 10.1111/bph.14454

13. Depriest AZ, Puet BL, Holt AC, Roberts A, Cone EJ. Metabolism and disposition of prescription opioids: a review. Forensic Sci Rev. (2015) 27:115-45.

14. Ishii Y, Miyoshi A, Watanabe R, Tsuruda K, Tsuda M, Yamaguchi-Nagamatsu Y, et al. Simultaneous expression of guinea pig UDP-glucuronosyltransferase 2B21 and 2B22 in COS-7 cells enhances UDP-glucuronosyltransferase 2B21-catalyzed morphine-6-glucuronide formation. Mol Pharmacol. (2001) 60:1040-8. doi: 10.1124/mol.60.5.1040

15. Kurita A, Miyauchi Y, Ikushiro SI, Mackenzie PI, Yamada H, Ishii Y. Comprehensive characterization of mouse UDP-glucuronosyltransferase (Ugt) belonging to the Ugt2b subfamily: identification of Ugt2b36 as the predominant isoform involved in morphine glucuronidation. J Pharmacol Exp Ther. (2017) 361:199-208. doi: 10.1124/jpet.117.240382

16. Zelcer N, van de Wetering K, Hillebrand M, Sarton E, Kuil A, Wielinga PR, et al. Mice lacking multidrug resistance protein 3 show altered morphine pharmacokinetics and morphine-6-glucuronide antinociception. Proc Natl Acad Sci USA. (2005) 102:7274-9. doi: 10.1073/pnas.0502530102

17. Beijnen JH, Schellens JH. Drug interactions in oncology. Lancet Oncol. (2004) 5:489-96. doi: 10.1016/S1470-2045(04)01528-1

18. Waters NJ. Evaluation of drug-drug interactions for oncology therapies: in vitro-in vivo extrapolation model-based risk assessment. Br J Clin Pharmacol. (2015) 79:946-58. doi: 10.1111/bcp.12563

19. Shiratani H, Katoh M, Nakajima M, Yokoi T. Species differences in UDPglucuronosyltransferase activities in mice and rats. Drug Metab Dispos. (2008) 36:1745-52. doi: 10.1124/dmd.108.021469

20. Mackenzie PI, $\mathrm{Hu}$ DG, Gardner-Stephen DA. The regulation of UDP-glucuronosyltransferase genes by tissue-specific and ligand-activated transcription factors. Drug Metab Rev. (2010) 42:99-109. doi: 10.3109/03602530903209544

21. Johanning J, Kroner P, Thomas M, Zanger UM, Norenberg A, Eichelbaum M, et al. The formation of estrogen-like tamoxifen metabolites and their influence on enzyme activity and gene expression of ADME genes. Arch Toxicol. (2018) 92:1099-112. doi: 10.1007/s00204-017-2147-y

22. Falany JL, Pilloff DE, Leyh TS, Falany CN. Sulfation of raloxifene and 4hydroxytamoxifen by human cytosolic sulfotransferases. Drug Metab Dispos. (2006) 34:361-8. doi: 10.1124/dmd.105.006551

23. Squirewell EJ, Qin X, Duffel MW. Endoxifen and other metabolites of tamoxifen inhibit human hydroxysteroid sulfotransferase 2A1 (hSULT2A1). Drug Metab Dispos. (2014) 42:1843-50. doi: 10.1124/dmd.114.059709

24. Tzvetkov MV, dos Santos Pereira JN, Meineke I, Saadatmand AR, Stingl JC, Brockmöller J. Morphine is a substrate of the organic cation transporter OCT1 and polymorphisms in OCT1 gene affect morphine pharmacokinetics after codeine administration. Biochem Pharmacol. (2013) 86:666-78. doi: 10.1016/j.bcp.2013.06.019

25. Iusuf D, Teunissen SF, Wagenaar E, Rosing H, Beijnen JH, Schinkel AH. Pglycoprotein $(\mathrm{ABCB} 1)$ transports the primary active tamoxifen metabolites endoxifen and 4-hydroxytamoxifen and restricts their brain penetration. $J$ Pharmacol Exp Ther. (2011) 337:710-7. doi: 10.1124/jpet.110.178301

26. Teft WA, Mansell SE, Kim RB. Endoxifen, the active metabolite of tamoxifen, is a substrate of the efflux transporter P-glycoprotein (multidrug resistance 1). Drug Metab Dispos. (2011) 39:558-62. doi: 10.1124/dmd.110.036160

27. Swarm RA, Paice JA, Anghelescu DL, Are M, Bruce JY, Buga S, et al. Adult cancer pain, Version 3.2019, NCCN clinical practice guidelines in oncology. J Natl Compr Cancer Netw. (2019) 17:977-1007. doi: 10.6004/jnccn.2019.0038
28. Vree TB, Verwey-van Wissen CP. Pharmacokinetics and metabolism of codeine in humans. Biopharm Drug Dispos. (1992) 13:445-60. doi: 10.1002/bdd.2510130607

29. Mutlib AE, Goosen TC, Bauman JN, Williams JA, Kulkarni S, Kostrubsky S. Kinetics of acetaminophen glucuronidation by UDP-glucuronosyltransferases 1A1, 1A6, 1A9 and 2B15. Potential implications in acetaminophen-induced hepatotoxicity. Chem Res Toxicol. (2006) 19:701-9. doi: 10.1021/tx05 $0317 \mathrm{i}$

30. Goss PE, Strasser K. Aromatase inhibitors in the treatment and prevention of breast cancer. J Clin Oncol. (2001) 19:881-94. doi: 10.1200/JCO.200 1.19.3.881

31. Linardi A, Damiani D, Longui CA. The use of aromatase inhibitors in boys with short stature: what to know before prescribing? Arch Endocrinol Metab. (2017) 61:391-7. doi: 10.1590/2359-3997000000284

32. Hasselstrom J, Sawe J. Morphine pharmacokinetics and metabolism in humans. Enterohepatic cycling and relative contribution of metabolites to active opioid concentrations. Clin Pharmacokinet. (1993) 24:34454. doi: 10.2165/00003088-199324040-00007

33. Handal M, Grung M, Skurtveit S, Ripel A, Morland J. Pharmacokinetic differences of morphine and morphine-glucuronides are reflected in locomotor activity. Pharmacol Biochem Behav. (2002) 73:88392. doi: 10.1016/S0091-3057(02)00925-5

34. Stearns V, Johnson MD, Rae JM, Morocho A, Novielli A, Bhargava P, et al. Active tamoxifen metabolite plasma concentrations after coadministration of tamoxifen and the selective serotonin reuptake inhibitor paroxetine. J Natl Cancer Inst. (2003) 95:1758-64. doi: 10.1093/jnci/djg108

35. MacLeod AK, McLaughlin LA, Henderson CJ, Wolf CR. Application of Mice Humanized for CYP2D6 to the study of tamoxifen metabolism and drugdrug interaction with antidepressants. Drug Metab Dispos. (2017) 45:1722. doi: $10.1124 / \mathrm{dmd} .116 .073437$

36. Prueksaritanont T, Chu X, Gibson C, Cui D, Yee KL, Ballard J, et al. Drug-drug interaction studies: regulatory guidance and an industry perspective. AAPS J. (2013) 15:629-45. doi: 10.1208/s12248-013-9470-x

37. Whitfield J, Littlewood T, Soucek L. Tamoxifen administration to mice. Cold Spring Harb Protoc. (2015) 2015:269-71. doi: 10.1101/pdb.prot077966

38. Shin SC, Choi JS, Li X. Enhanced bioavailability of tamoxifen after oral administration of tamoxifen with quercetin in rats. Int J Pharm. (2006) 313:144-9. doi: 10.1016/j.ijpharm.2006.01.028

39. Buchanan CM, Buchanan NL, Edgar KJ, Little JL, Malcolm MO, Ruble KM, et al. Pharmacokinetics of tamoxifen after intravenous and oral dosing of tamoxifen-hydroxybutenyl-beta-cyclodextrin formulations. J Pharm Sci. (2007) 96:644-60. doi: 10.1002/jps.20709

40. Undevia SD, Gomez-Abuin G, Ratain MJ. Pharmacokinetic variability of anticancer agents. Nat Rev Cancer. (2005) 5:447-58. doi: 10.1038/nrc1629

Conflict of Interest: The authors declare that the research was conducted in the absence of any commercial or financial relationships that could be construed as a potential conflict of interest.

Copyright (c) 2020 Gabel, Aubry, Hovhannisyan, Chavant, Weinsanto, Maduna, Darbon and Goumon. This is an open-access article distributed under the terms of the Creative Commons Attribution License (CC BY). The use, distribution or reproduction in other forums is permitted, provided the original author(s) and the copyright owner(s) are credited and that the original publication in this journal is cited, in accordance with accepted academic practice. No use, distribution or reproduction is permitted which does not comply with these terms. 